\title{
Kinetic modeling of the electric double layer at a dielectric plasma-solid interface
}

\author{
K. Rasek, F. X. Bronold and H. Fehske \\ Institut für Physik, Universität Greifswald, 17489 Greifswald, Germany
}

(Dated: April 16, 2020)

\begin{abstract}
For a collisionless plasma in contact with a dielectric surface, where with unit probability electrons and ions are, respectively, absorbed and neutralized, thereby injecting electrons and holes into the conduction and valence band, we study the kinetics of plasma loss by nonradiative electron-hole recombination inside the dielectric. We obtain a self-consistently embedded electric double layer, merging with the quasi-neutral, field-free regions inside the plasma and the solid. After a description of the numerical scheme for solving the two sets of Boltzmann equations, one for the electrons and ions of the plasma and one for the electrons and holes of the solid, to which this transport problem gives rise to, we present numerical results for a p-doped dielectric. Besides potential, density, and flux profiles, plasma-induced changes in the electron and hole distribution functions are discussed, from which a microscopic view on plasma loss inside the dielectric emerges.
\end{abstract}

\section{INTRODUCTION}

At the interface between a low-temperature plasma and a macroscopic solid an electric double layer forms consisting of a plasma-bound electron-depleted and a solid-bound electron-rich region. In the simplest scenario, the charge separation arises because electrons, outrunning ions on the plasma side, are more efficiently deposited into the surface than they are extracted from it by the neutralization of ions which effectively leads to the injection of missing electrons, that is, in the language of solid state physics to the injection of holes. At the end a potential profile builds up equalizing the electron and ion fluxes issued by the plasma source with the electron-hole recombination flux inside the solid. The double layer is hence caused by the plasma but controlled by the solid.

Little is quantitatively known about the scenario although the positive part of the double layer-the plasma sheath-has been studied in great detail ever since the work by Langmuir and Mott-Smith [1]. Most of the studies focus on the merging of the sheath with the quasineutral bulk plasma [2 6 ] . The effect of the solid is studied only in as far as its emissive properties, electron/ion reflection and secondary electron emission, affect the stability of the sheath $7-12$. The reasoning behind it is the assumption that processes inside the solid occur on spatio-temporal scales too small or too fast to affect the physics of the plasma [13]. For the plasma species the solid is thus only a sink or source characterized by probabilities for absorption, reflection and emission which, in principle, can be measured [14 16] or calculated 1719]. There are however also theoretical approaches 2023] treating the solid and the plasma as two sides of an interface to be analyzed together.

Mapping the charge dynamics of the solid in contact with the plasma to a set of parameters is no longer justified in situations where the scales of the plasma and the solid become comparable or where the solid is an integral part of the plasma device of interest as it is, for instance, the case in attempts to combine gaseous with solid state electronics 24 28]. In particular, if the miniaturization of the devices continues [29], the transit times through the plasma and the transport and relaxation times inside the solid may become comparable, requiring then to resolve the charge dynamics inside the solid and the plasma at the same kinetic level.

Recently, we set up a theoretical framework showing how such a calculation can be organized for a plasmafacing dielectric solid [21]. It is based on two sets of spatially separated Boltzmann equations, one for the electrons and ions inside the plasma and one for the conduction band electrons and valence band holes inside the dielectric. The two sets are coupled by the electric field, entering the force terms of the Boltzmann equations and being the solution of the Poisson equation, and matching conditions at the interface describing electron transmission and reflection in either way as well as hole injection due to the neutralization of ions. To demonstrate the feasibility of the approach, we applied it to a collisionless, perfectly absorbing interface with an ad-hoc recombination condition to prevent-in a collisionless situationthe unlimited growth of the charge carriers inside the solid. Although conceptually incomplete at this point, it seemed useful because a numerical solution of the Boltzmann equation could be avoided.

The purpose of the present work is to remedy this shortcoming by applying the theoretical framework to an interface which is left collisionless only on the plasma side, where it can be justified, because electrons are strongly depleted, scattering hence only weakly, while ions collide predominantly with neutrals, which is important only in particular situations [30 32]. But by including collisions on the solid side, we can now couple the creation of charge carriers by the plasma source to the physical process destroying them inside the solid. It is the balancing of the two at quasi-stationarity which determines quantitatively the charge and potential profiles on both sides of the interface.

The paper is structured as follows. In section II divided into two subsections, we present in $\amalg$ A a simplified kinetic model for the double layer at a dielectric plasmasolid interface and in IB the numerical strategy for its solution. Energy and momentum relaxation due to scattering on optical phonons [33] and nonradiative electron- 
hole recombinations due to traps in the energy gap along the lines of a kinetic version [34] of the Shockley-ReadHall model [35, 36] are taken into account by the kinetic equations while charge injection is treated phenomenologically by source functions entering the boundary conditions. The numerical approach utilizes an idea of Grinberg and Luryi [37] for solving iteratively Boltzmann equations with distribution functions known at the two end points of the integration domain, successfully applied to solid-solid interfaces 38 40]. Its utility in the present context is based on the observation that at the interface the distribution functions can be assumed to be known from the previous iteration loop and successively updated until convergence is reached. Combined with the boundary conditions fixing the distribution functions deep inside the solid and the plasma, a transport problem arises to which the Grinberg-Luryi approach can be applied in each half-space. Care is however required for treating singular points arising either from turning points or the vanishing of the electric field due to the embedding between field-free bulk regions. Numerical results are given in section \II for a p-doped dielectric. Potential, charge density, and flux profiles are shown together with the distribution functions for the dielectric's surplus carriers originating from the plasma. Section IV concludes the presentation and mathematical details are provided in three appendices.

\section{THEORY}

The notation used for the description of the electric double layer at a floating dielectric plasma-solid interface is summarized in Fig. 1. Also shown is the simplification required due to numerical constraints, forcing us to restrict the modeling on the solid side to the region close to the band edges. The injection of charge carriers into the solid has thus to be taken into account by phenomenological source functions.

\section{A. Formulation of the transport problem}

Within the coordinate system of Fig. 1 the plasmasolid interface is located at $z=0$ with the solid and plasma filling up the halfspaces $z<0$ and $z>0$. The interface is abrupt with material parameters constant and isotropic within each halfspace. The spatial dependencies arise from the electric potential energy $U_{c}(z)$ for which we set $U_{c}(0)=0$. The merging of the double layer with the quasi-neutral, field-free regions occurs at $z_{1}$ on the solid side and at $z_{p}$ on the plasma side. Since the solid and the plasma accumulate net negative and positive charge, respectively, $U_{c}(z)$ is monotonously increasing with $z$.

Instead of $z$ we can thus use $U_{c}$ to track the spatial dependency of all physical quantities, with the mapping between the two given by the once-integrated Poisson

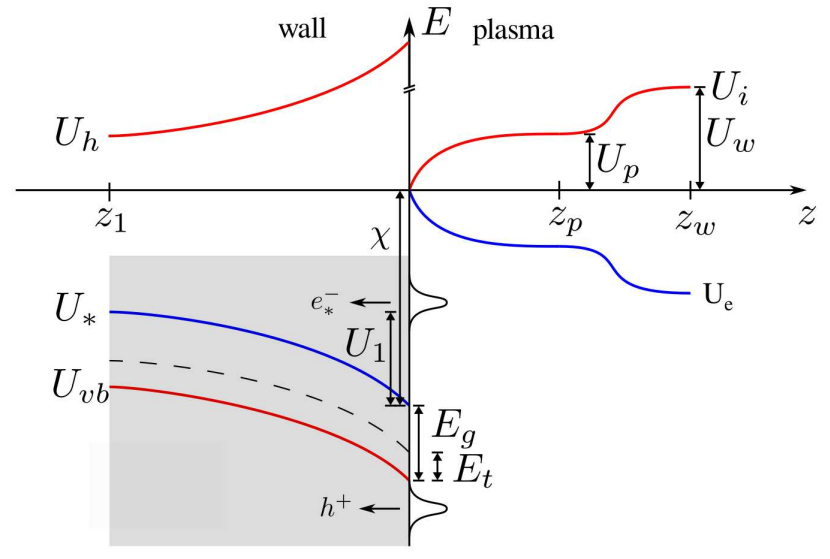

FIG. 1. Illustration of the potential energy profiles for an electric double layer at a floating dielectric plasma-wall interface (not on scale). Shown are the edges of the conduction $\left(U_{*}\right)$ and valence $\left(U_{v b}\right)$ bands, the edge for the motion of valence band holes $\left(U_{h}\right)$, the position of the trap levels $\left(E_{t}\right)$, and the potential energy for electrons $\left(U_{e}\right)$ and ions $\left(U_{i}\right)$ on the plasma side. The origin of the energy axis is the potential just out-side the solid, $\chi$ is the electron affinity, and $E_{g}$ the energy gap. The positions $z_{1}$ and $z_{p}$ are the end points of the double layer and $z_{w}$ is the location of the plasma source. Also shown are the potential energies at these positions, playing an important role in the modeling. As explained in the main text, we cannot resolve the energy space up to the energies where carriers are actually injected from the plasma into the solid. In the simplified model for the solid side, shown in dark, we inject carriers at artificially low energies by source functions included in the matching conditions at $z=0$.

equation,

$$
\frac{\mathrm{d} U_{c}}{\mathrm{~d} z}=\left(\frac{16 \pi}{\varepsilon(z)} \int_{U_{0}(z)}^{U_{c}(z)} \mathrm{d} U n(U)\right)^{1 / 2}=\mathcal{E}\left(U_{c}\right)
$$

where $\varepsilon(z)=\Theta(z)+\varepsilon \Theta(-z)$, with $\Theta(z)$ the usual step function, is the dielectric function of the interface. The function $U_{0}(z)=U_{p} \Theta(z)+U_{1} \Theta(-z)$ denotes the potential energy, where the net charge vanishes, that is, where quasi-neutrality holds. On the solid side, this is at $z=z_{1}$ leading to $U_{c}=U_{1}$, where $U_{1}<0$ is the band bending, while on the plasma side it occurs at $z=z_{p}$ and hence at $U_{c}=U_{p}>0$. The function $\mathcal{E}(z)$ is the (negative) electric field for which

$$
\varepsilon \mathcal{E}\left(0^{-}\right)=\mathcal{E}\left(0^{+}\right)
$$

holds at the interface and the (negative) total charge to be integrated over reads for a p-doped interface

$$
\begin{aligned}
n\left(U_{c}\right)=\left[n_{e}\left(U_{c}\right)-n_{i}\left(U_{c}\right)\right] \Theta\left(U_{c}\right) & \\
& +\left[n_{*}\left(U_{c}\right)-n_{h}\left(U_{c}\right)+n_{A}\right] \Theta\left(-U_{c}\right),
\end{aligned}
$$

where $n_{A}$ is the concentration of the acceptors. 
Introducing a species index $s \in\{i, e, h, *\}$ for ions, electrons, valence band holes, and conduction band electrons, the potential energy $U_{s}$ can be defined for each species. Its relation to $U_{c}$ is given by the following expressions, taking into account the energy offsets of Fig 1. $U_{i}=U_{c}, U_{e}=-U_{c}, U_{h}=U_{c}+E_{g}+\chi$, and $U_{*}=-U_{c}-\chi$, where $\chi$ is the electron affinity and $E_{g}$ is the band gap of the dielectric. We will use $U_{s}$ as a variable synonymous to $U_{s}\left(U_{c}\right)$.

It is advantageous to introduce in the coordinate system of Fig. 1 separate distribution functions, $F_{s}^{<}$and $F_{s}^{>}$, for the left- and right-moving particles with the sign of the perpendicular momentum $k_{z}$ encoded in the superscript. Since the interface is homogeneous in the lateral directions it is also rotationally invariant in the plane perpendicular to the $z$ axis. The distribution functions depend thus only on the magnitude of the lateral momentum $\mathbf{K}$. Instead of it, we use the lateral kinetic energy $T=\hbar^{2} \mathbf{K}^{2} / 2 m_{s}$ as a variable, where $m_{s}$ is the mass of a particle of species $s$. In atomic units, measuring length in Bohr radii, energy in Rydbergs, and mass in electron masses, the Boltzmann equation can be cast into

$$
\pm v_{s}\left(U_{c}, E, T\right) \mathcal{E}\left(U_{c}\right) \frac{\partial}{\partial U_{c}} F_{s}^{\gtrless}\left(U_{c}, E, T\right)=I_{\text {coll }}^{\gtrless},
$$

where $k_{z}$ is replaced by the total energy $E, I_{\text {coll }}^{\gtrless}$ is the collision integral, and

$$
v_{s}\left(U_{c}, E, T\right)=2 \sqrt{m_{s}^{-1}\left(E-U_{s}-T\right)}
$$

is the velocity perpendicular to the interface. Due to the variable transformation (3) from $z$ to $U_{c}$ the force term in (4) accounts automatically for the Poisson equation.

The collision integral $I_{\text {coll }}^{\gtrless}$, describing scattering and recombination processes, depends on either side of the interface on the distribution functions of both species. It can be separated into in- and out-scattering parts, $\Phi_{s}^{\gtrless}$ and $\gamma_{s}^{\gtrless} F_{s}^{\gtrless}$, respectively, turning the Boltzmann equation (44) into its final form,

$$
\pm v_{s}\left(U_{c}\right) \mathcal{E}\left(U_{c}\right) \frac{\partial}{\partial U_{c}} F_{s}^{\gtrless}\left(U_{c}\right)=\Phi_{s}^{\gtrless}\left(U_{c}\right)-\gamma_{s}^{\gtrless}\left(U_{c}\right) F_{s}^{\gtrless}\left(U_{c}\right) \text {, }
$$

where we have omitted the dependencies on $E, T$, and $F_{s}^{\gtrless}$. In appendix A we give $\Phi_{s}^{\gtrless}$ and $\gamma_{s}^{\gtrless}$ for scattering on optical phonons [33] and recombination via traps in the energy gap [34], which is a kinetic formulation of the Shockley-Read-Hall model [35, 36].

Once the solutions of (6) are known, the densities

$$
n_{s}\left(U_{c}\right)=\frac{m_{s}}{8 \pi^{2}} \int \mathrm{d} E \mathrm{~d} T \frac{F_{s}^{>}\left(U_{c}, E, T\right)+F_{s}^{<}\left(U_{c}, E, T\right)}{v_{s}\left(U_{c}, E, T\right)},
$$

can be obtained, from which the electric field $\mathcal{E}\left(U_{c}\right)$ follows by iterating (11), closing thereby the set of equations.

An essential part of the transport problem are the boundary conditions at $U_{c}=U_{1}$ and $U_{c}=U_{w}$ and the matching condition at $U_{c}=0$. The boundary conditions are given by

$$
\begin{array}{cl}
F_{s}^{>}\left(U_{1}\right)=F_{s}^{\mathrm{LM}}\left(U_{1}\right) & \text { for } s=h, *, \\
F_{s}^{<}\left(U_{w}\right)=F_{s}^{\mathrm{LM}}\left(U_{w}\right) & \text { for } s=i, e
\end{array}
$$

with

$$
F_{s}^{\mathrm{LM}}\left(U_{c}\right)=n_{s}^{\mathrm{LM}}\left(\frac{4 \pi}{k_{B} T_{s} m_{s}}\right)^{3 / 2} \exp \left(-\frac{E-U_{s}}{k_{B} T_{s}}\right)
$$

a half-Maxwellian with temperature $T_{s}$ and density $n_{s}^{\mathrm{LM}}$.

The general matching conditions for the distribution functions at $U_{c}=0$ are given in Ref. [21]. We specialize them now to an interface, where electrons can pass the interface only from the plasma side and ions are neutralized at the interface with unit probability. Carriers approaching the interface from the solid side are specularly reflected.

Anticipating the potential energy profile of a double layer with negative and positive net charge inside the solid and the plasmas, respectively, the matching conditions for the electron distribution functions read

$$
\begin{aligned}
F_{e}^{>}(0, E, T) & =0 \text { for } E>0, \\
F_{e}^{>}\left(U_{c}, E, T\right) & =F_{e}^{<}\left(U_{c}, E, T\right) \text { for } E=U_{e}, \\
F_{*}^{<}(0, E, T) & =F_{*}^{>}(0, E, T)+S_{*}^{<}(0, E, T),
\end{aligned}
$$

while for the ion and hole distribution functions they become

$$
\begin{aligned}
& F_{i}^{>}(0, E, T)=0, \\
& F_{h}^{<}(0, E, T)=F_{h}^{>}(0, E, T)+S_{h}^{<}(0, E, T),
\end{aligned}
$$

where we introduced source functions encoding electron and hole injection,

$$
\begin{aligned}
S_{s}^{<}\left(U_{s}, E, T\right) & =n_{s}^{\text {in }}\left(\frac{4 \pi}{k_{B} T_{s} m_{s}}\right)^{\frac{3}{2}} \\
& \times \exp \left(-\frac{\left(E-U_{s}-I_{s}^{\text {in }}\right)^{2}-T^{2}}{\Gamma_{\text {in }}^{2}}\right),
\end{aligned}
$$

with injection densities $n_{s}^{\text {in }}$ chosen such that $j_{h}^{\text {in }}=j_{i}$ and $j_{*}^{\text {in }}=j_{e}$. The electron and ion fluxes from the plasma, $j_{e}$ and $j_{i}$, are given by

$$
j_{s}\left(U_{c}\right)=m_{s} \int \frac{\mathrm{d} E \mathrm{~d} T}{8 \pi^{2}}\left[F_{s}^{>}\left(U_{c}, E, T\right)-F_{s}^{<}\left(U_{c}, E, T\right)\right],
$$

and $j_{s}^{\text {in }}$ is obtained from (17) by setting $F_{s}^{>}=0$ and $F_{s}^{<}=S_{s}^{<}$. For simplicity we take phenomenological Gaussians with width $\Gamma_{\text {in }}$ centered around $E-U_{s}=I_{s}^{\text {in }}$ and $T=0$ as source functions.

Ideally, the injection energies $I_{s}$ would be the real ones, set by the ion's ionization energy, in case of resonant ionization, and the dielectric's electron affinity. Both are usually a couple of $\mathrm{eV}$ away from the band edges. The 
relaxation and recombination kinetics, on the other hand, making at the end the space charge inside the solid quasistationary with the plasma sheath, requires a resolution on the order of the phonon energy, which is typically $0.1 \mathrm{eV}$. Resolving on that scale the whole energy range up to the actual injection points is computationally very expensive. To keep the numerical costs at an acceptable level, we move the injection energies $I_{s}$ below an energy cutoff dictated by numerical constraints. The principal mechanism of the model, relaxation and subsequent recombination of plasma-injected surplus charges inside the plasma-facing solid, remains intact.

\section{B. Numerical strategy}

We now sketch the numerical approach employed to solve the transport problem, focusing on the overall strategy to determine the various parameters required to selfconsistently embed the double layer between field-free, quasi-neutral bulk regions. Technical details concerning the plasma side and the integration routines are relegated to appendices $\mathrm{B}$ and $\mathrm{C}$

The plasma source issues at $U_{c}=U_{w}$ ions and electrons belonging to half-Maxwellians characterized by $T_{e, i}$ and $n_{e, i}^{\mathrm{LM}}$. Input parameters are only the temperatures. The densities are determined from the model in a two-step procedure. First, enforcing the absence of an electric field and the quasi-neutrality at $U_{c}=U_{p}<U_{w}$, giving rise to the two conditions,

$$
\begin{aligned}
& \mathcal{E}\left(U_{p}\right)=0, \\
& n\left(U_{p}\right)=0,
\end{aligned}
$$

and combining them with the flux equality,

$$
j_{e}\left(U_{c}\right)=j_{i}\left(U_{c}\right),
$$

the density ratio $\alpha=n_{i}^{\mathrm{LM}} / n_{e}^{\mathrm{LM}}$, to be interpreted as the strength of the plasma source, and the two potential parameters $U_{p}$ and $U_{w}$ can be determined. In a second step, the matching (2) of the electric field across the interface, feeding in information form the solid side, is used to determine the absolute values of $n_{e}^{\mathrm{LM}}$ and $n_{i}^{\mathrm{LM}}$.

On the solid side, we use half-Maxwellians at $U_{c}=U_{1}$. The temperatures characterizing them are again input parameters, while the densities are determined by the absence of an electric field and the quasi-neutrality, yielding the three conditions

$$
\begin{aligned}
\mathcal{E}\left(U_{1}\right) & =0, \\
n\left(U_{1}\right) & =0, \\
n_{h}^{\mathrm{LM}} n_{*}^{\mathrm{LM}} & =n_{\text {int }}^{2}
\end{aligned}
$$

with the intrinsic density

$$
n_{\text {int }}=\frac{1}{4}\left(\frac{k_{B} T_{*}}{\pi}\right)^{3 / 2}\left(m_{*} m_{h}\right)^{3 / 4} \exp \left(-\frac{E_{g}}{2 k_{B} T_{*}}\right),
$$

TABLE I. The two sets of material parameters (a) and (b) we used in our numerical calculations. For the Debye lengths the acceptor density $n_{A}$ is used instead of the intrinsic density $n_{\text {int }}$. For set (b) only values different from set (a) are displayed.

\begin{tabular}{lll}
\hline \hline & $(\mathrm{a})$ & $(\mathrm{b})$ \\
\hline$E_{g}[\mathrm{eV}]$ & 1 & 2 \\
$\hbar \omega_{0}[\mathrm{meV}]$ & 75 & \\
$E_{t}[\mathrm{eV}]$ & 0.4 & 0.3 \\
$\varepsilon$ & 11.8 & \\
$\varepsilon_{\infty}$ & 12 & $10^{14}$ \\
$n_{A}\left[\mathrm{~cm}^{-3}\right]$ & $10^{13}$ & $10^{-8}$ \\
$n_{\mathrm{int}}\left[10^{10} \mathrm{~cm}^{-3}\right]$ & 4.922 & \\
$N_{t}\left[\mathrm{~cm}^{-3}\right]$ & $10^{20}$ & \\
$\sigma_{s}\left[\mathrm{~cm}^{2}\right]$ & $10^{-15}$ & \\
$k_{B} T_{*, h}[\mathrm{eV}]$ & 0.025 & 0.576 \\
$m_{*, h}\left[m_{e}\right]$ & 1 & \\
$\lambda_{D}^{w}[\mu \mathrm{m}]$ & 1.821 & \\
\hline \hline
\end{tabular}

where we set $T_{*}=T_{h}$. From the three equations the two densities $n_{*}^{\mathrm{LM}}$ and $n_{h}^{\mathrm{LM}}$ as well as the band bending $U_{1}$ can be determined. The parameters of the source functions $S_{*, h}^{<}$are either input parameters $\left(I_{*, h}^{\text {in }}, \Gamma_{\text {in }}\right)$ or fixed by flux continuity $\left(n_{*, h}^{\text {in }}\right)$. All free parameters are thus determined and the double layer is selfconsistently embedded between the two quasi-neutral, field-free regions.

Due to the collisionality, the modeling on the solid side requires only one potential energy parameter, the band bending $U_{1}$. The distribution functions $F_{*, h}^{\gtrless}\left(U_{c}, E, T\right)$ can be taken as half-Maxwellians at $U_{c}=U_{1}$ because the vanishing of the field makes them in (6) to annihilate the collision integrals. With half-Maxwellians, satisfying detailed balance, this can be enforced. On the plasma side, however, being collisionless, the distribution functions cannot be half-Maxwellians at $U_{c}=U_{p}$. They have to be put in at $U_{c}=U_{w}>U_{p}$ by the Schwager-Birdsall construction [2] leading to two potential energy parameters, $U_{p}$ and $U_{w}$.

In order to get the density and potential profiles to be employed in the embedding conditions just listed, the Boltzmann equation (6) has to be solved. On the plasma side this can be done analytically. Following the approach of Schwager and Birdsall [2], it leads to the expressions listed in appendix B. Had we also included collisions there, a numerical solution along the lines we now present for the solid side would be in order.

The numerical approach for solving the Boltzmann equations for electrons and holes inside the dielectric is an iterative scheme, originally proposed by Grinberg and Luryi [37] for transport problems where distribution functions are known at the two end points of the integration domain. It has proven its feasibility for solid-solid interfaces [38 40] and can be based on a rewriting of the Boltzmann equation (6) for right- and left-moving distri- 
butions in the form (in the following $s=*, h$ )

$$
\begin{aligned}
F_{s}^{>}\left(U_{c}\right) & =\xi_{s}\left(U_{c}, U_{c}-\Delta\right) F_{s}^{>}\left(U_{c}-\Delta\right) \\
& +\int_{U_{c}-\Delta}^{U_{c}} \frac{\mathrm{d} U}{\mathcal{E}(U)} \frac{\Phi_{s}^{>}(U)}{v_{s}(U)} \xi_{s}\left(U_{c}, U\right)
\end{aligned}
$$

and

$$
\begin{aligned}
F_{s}^{<}\left(U_{c}\right) & =\xi_{s}\left(U_{c}+\Delta, U_{c}\right) F_{s}^{<}\left(U_{c}+\Delta\right) \\
& +\int_{U_{c}}^{U_{c}+\Delta} \frac{\mathrm{d} U}{\mathcal{E}(U)} \frac{\Phi_{s}^{<}(U)}{v_{s}(U)} \xi_{s}\left(U, U_{c}\right)
\end{aligned}
$$

with the integrating factor

$$
\xi_{s}\left(U_{c}, U_{c}^{\prime}\right)=\exp \left(-\int_{U_{c}^{\prime}}^{U_{c}} \frac{\mathrm{d} U}{\mathcal{E}\left(\bar{U}_{c}\right)} \frac{\gamma_{s}^{\gtrless}(U)}{v_{s}(U)}\right),
$$

where $\Delta$ is an arbitrary energy shift, but at the end it will be the basic discretization step in $U_{c^{-}}$direction. The two equations are an exact rewriting of the original Boltzmann equations utilizing (i) the fact that in the variable $U_{c}$ they are ordinary first order differential equations and (ii) that the integrating factor $\xi_{s}\left(U_{c}, U_{c}^{\prime}\right)$ satisfies group properties. For brevity, the $E$ and $T$ dependencies of the various functions are again suppressed.

The iteration scheme we employed for solving Eqs. (25) and (26) is illustrated in Fig. 7 of appendix [C] To obtain the distribution function $F_{s}^{>}\left(U_{c}\right)$ in the interval $U_{1}<U_{c}<0$, Eq. (25) is iterated from $U_{c}=U_{1}$ to $U_{c}=0$, while $F_{s}^{<}\left(U_{c}\right)$ is obtained from (26) by iterating it from $U_{c}=0$ to $U_{c}=U_{1}$, using at the starting points the boundary and matching conditions specified above, and in the collision integrals the distribution functions of the previous iteration loop. Special care has to be exercised by the discretization of the integrals near singular points, where the left hand side of (6) vanishes, leading to singularities in the integrals. Due to the collisions encoded in the functions $\gamma_{s}^{\gtrless}\left(U_{c}\right)$ and $\Phi_{s}^{\gtrless}\left(U_{c}\right)$ the variables $E$ and $T$ are not spectators of the integration procedure, as the simplified notation of (25) and (26) may suggest. In total, we have to iterate in a three-dimensional domain spanned by the variables $U_{c}, E$, and $T$. Further details and delicacies of the integration routines are discussed in appendix $\mathrm{C}$

\section{RESULTS}

We now apply our model to a p-doped dielectric plasma-solid interface characterized by the parameters of Table I Although we do not attempt to describe specific materials in contact with specific plasmas, the parameters are chosen to represent a typical semiconductor facing a hydrogen plasma. The parameters of the source functions (16), determined by the continuity of fluxes at $U_{c}=0$, are summarized in Table [1] while the specifics of the plasma are given in Table III. The values of the potential energy $U_{c}$ at $z=z_{1}, z_{p}, z_{w}$, denoting respectively the band bending, the sheath potential, and the drop of the sheath of the plasma source, listed in Table IV] are no input parameters. They arise from the selfconsistent matching of the solid and the plasma.

The trap density $N_{t}$ in Table $\square$ is artificially high because the coordinate transformation (10), mapping an infinite $z$-halfspace to a finite $U_{c}$-interval, restricts de facto the modeling to the region where the band bending is significant. In general, this is favorable. But for the recombination process it is a problem since the recombination length $\lambda_{R}$, given in a rough approximation by

$$
\lambda_{R} \approx \frac{v_{s}}{\gamma_{s}^{\text {trap }}} \approx \frac{1}{\sigma_{s} N_{t}},
$$

is for the realistic cross section $\sigma_{s} \approx 10^{-15} \mathrm{~cm}^{2}$ and the realistic trap density $N_{t} \approx 10^{16} \mathrm{~cm}^{-3}$ too large. It is on the order of $m m$ while the Debye length $\lambda_{D}^{w}$, setting the scale of the space charge layer, and hence of our simulation domain, is only a few $\mu \mathrm{m}$. To ensure complete recombination in the numerically resolved domain, necessary to prevent a pile-up of charges inside the solid, we have to increase thus $N_{t}$ by four orders of magnitude.

Let us start the discussion of the numerical data with the selfconsistent electric fields shown in Fig. 2 for parameter sets (a) and (b). The matching condition (2) is satisfied for both sets. Due to the higher acceptor concentration of set (b), the Debye length is shorter yielding a narrower space charge and a smaller band bending. The large fields on the solid side are in both cases mainly caused by the charge carriers due to the doping and not due to the surplus carriers coming from the plasma. On the plasma side, the field is due to the sheath in front of the solid. The Schwager-Birdsall boundary condition [2] leads to the non-monotonous behavior around $U_{c} \approx 6.8 \mathrm{eV}$. It is an artifact arising from the inflection point in the potential profile required to model in a collisionless plasma a field-free, quasi-neutral region representing the bulk plasma. Due to the coordinate transformation (10), the range of $U_{c}$ values shown in the plot corresponds essentially to two infinite halfspaces in the variable $z$. Mapping an infinite system to a finite one is an advantage of the change of coordinates.

Having found selfconsistent embeddings of the double layer, we now turn to the distribution functions for elec-

TABLE II. Parameters of the source functions $S_{*}^{<}$and $S_{h}^{<}$describing the injection of electrons and holes from the plasma into the solid. The width $\Gamma^{\text {in }}$ is fine-tuned to match (2) exactly even for the finite resolution of the energy scales preventing the band bending $U_{1}$ to be specified to arbitrary precision.

\begin{tabular}{lll}
\hline \hline$\Gamma^{\text {in }}[\mathrm{eV}]$ & $I_{*}^{\text {in }}[\mathrm{eV}]$ & $I_{h}^{\text {in }}[\mathrm{eV}]$ \\
\hline 0.06 & 0.2 & 0.15 \\
\hline \hline
\end{tabular}




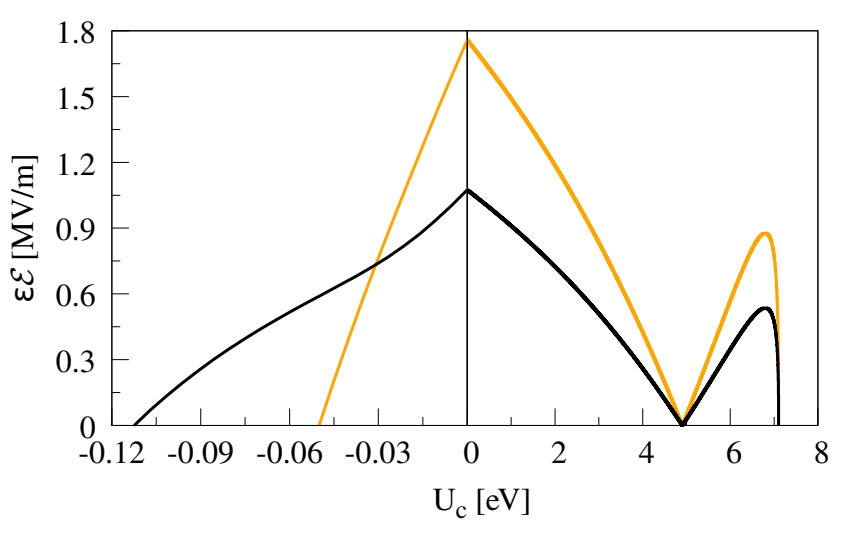

FIG. 2. Electric field multiplied by the dielectric function across the interface for the parameter sets (a) and (b), plotted respectively in black and orange, showing the matching (2) to be satisfied. The maximum at $U_{c} \approx 6.8 \mathrm{eV}$ comes from the Schwager-Birdsall boundary condition. Since $U_{p} \approx 5 \mathrm{eV}$ effectively corresponds already to $z \approx \infty$ the maximum has no physical meaning. It is an artifact of implementing in a collisionless plasma a field-free, quasi-neutral bulk region.

trons and holes inside the solid. Without plasma the electron and hole distribution functions are to a very good approximation half-Maxwellians, determined by the intrinsic carriers and the doping. Once the solid is in contact with the plasma, the distribution functions deviate from it due to the injection of carriers from the plasma and the band bending in response to the sheath potential. Since the two parameters sets yield rather similar results, we discuss below only data for one set.

Figure 3 shows for parameter set (a) the deviations of the distribution functions from the half-Maxwellian background directly at the interface at $U_{c}=0$ and inside the solid at $U_{c}=21.5 \mathrm{meV}$. To visualize physical effects more clearly, we plot $F_{*, h}^{\gtrless}$ for the two fixed values of $U_{c}$ as functions of $T_{z}=E-U_{*, h}-T$ and $E-U_{*, h}$, with $U_{*}=-\chi-U_{c}, U_{h}=U_{c}+E_{g}+\chi$, and $E$ running from $U_{s}$ to $E_{\max }=0.4 \mathrm{eV}$. Besides the peak at $E-U_{s}=I_{s}^{\text {in }}$ due to the source functions, clearly seen in the data for $U_{c}=0$, three further features can be identified: First, there is a series of peaks due to the scattering of the injected carriers on phonons. This is the energy and momentum relaxation of the carriers following injection from the plasma. Second, there is a step at $T_{z}=0$, separating the distributions for left- $\left(T_{z}<0\right)$ and

TABLE III. Parameters of the collisionless hydrogen plasma in contact with the dielectrics (a) and (b) specified in Table $\square$ For set (b) only values different from (a) are listed.

\begin{tabular}{llllll}
\hline \hline system & $k_{B} T_{e}[\mathrm{eV}]$ & $k_{B} T_{i}[\mathrm{eV}]$ & $m_{e}\left[m_{e}\right]$ & $m_{i}\left[m_{e}\right]$ & $\lambda_{D}^{p}[\mu \mathrm{m}]$ \\
\hline (a) & 0.025 & 2 & 1 & 1836 & 9.107 \\
(b) & & & & & 5.562 \\
\hline \hline
\end{tabular}

right-moving $\left(T_{z}>0\right)$ carriers (encoded in the artificial sign of $T_{z}$ ). Because left-moving distributions have to be populated by backscattering events, which for interaction with optical phonons are rather unlikely, they are always smaller than the right-moving distributions populated by forward scattering. Third, the functions are maximal for $E-U_{s} \approx 0$ since the carriers accumulate at the band edges.

That there are less right- than left-moving injected carriers can be also seen in Fig. 4, where we plot for the parameter set (a) the directional electron and hole densities scaled to the reference densities given in the caption. The densities have been calculated from the distribution functions $F_{*, h}^{\gtrless}$ using (77) and subtracting from them the background densities due to the doping. All the surplus densities are maximal at $U_{c}=0$, that is, directly at the interface, and monotonously decrease to zero by approaching the bulk of the solid. In the inset the difference of the densities of left- and right-moving carriers is shown. It is positive and of the same order for both polarities showing that both types of surplus carriers move preferentially to the left. From the plot we also see that injected electrons dominate injected holes as it should be for a double layer, where the positive, electron-depleted branch residing in front of the solid at $U_{c}>0$ has to be balanced by a net negative space charge inside the solid at $U_{c}<0$. The profiles demonstrate also that at quasistationarity the permanent influx of electrons and holes from the plasma does not lead to a pile-up of carriers inside the solid. Carrier recombination prevents this.

The net density and potential profiles of the double layer as a whole, embracing the solid and the plasma side, are shown in Fig. 5 as a function of $z$ scaled to the corresponding Debye lengths. Since the results are similar for the two parameter sets we show again only data for set (a). Due to the difference in the screening lengths, $\lambda_{D}^{w} \approx 1.8 \mu \mathrm{m}$ and $\lambda_{D}^{p}=9.107 \mu \mathrm{m}$ (see Tables $\Pi$ and III), the charge neutrality of the double layer is not directly obvious but indeed satisfied due to the matching condition (2) which also gives rise to the different slopes of the potential energy profile for $z=0^{-}$and $z=0^{+}$. The spatial scale of the double layer is set by the screening lengths. From the numerical values given in Tables [ and [II] we see that for both parameter sets the width is on the order of $1-10 \mu \mathrm{m}$ with the plasma side five- [set (a)] to ten-times [set (b)] thicker than the solid side. Notice the fast and slow decay of the density profile on the solid side in contrast to the more or less homogeneous decay

TABLE IV. Numerical values of the selfconsistently determined potential energies at $z=z_{1}, z=z_{p}$, and $z=z_{w}$ for the parameter sets (a) and (b) of Tables \and III Again, for (b) only values different from (a) are given.

\begin{tabular}{llll}
\hline \hline system & $U_{1}[\mathrm{eV}]$ & $U_{p}[\mathrm{eV}]$ & $U_{w}[\mathrm{eV}]$ \\
\hline (a) & 0.1125 & 4.906 & 7.103 \\
(b) & 0.05 & & \\
\hline \hline
\end{tabular}




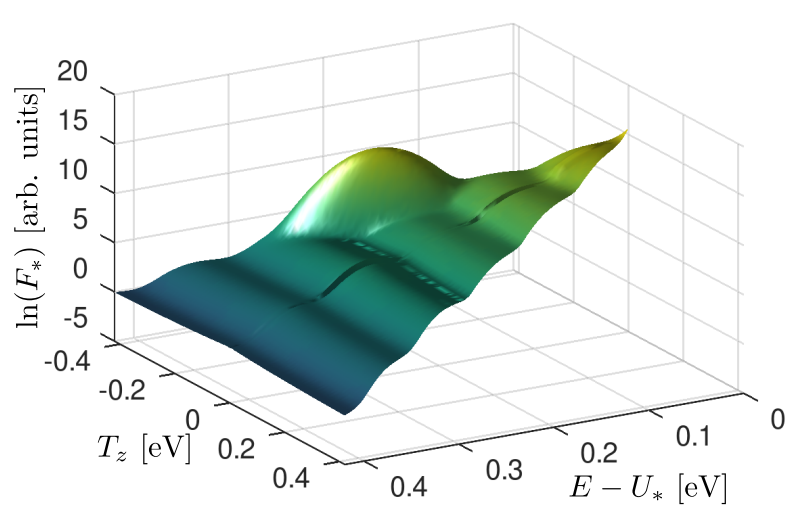

(a) Electron distribution at $U_{c}=0$

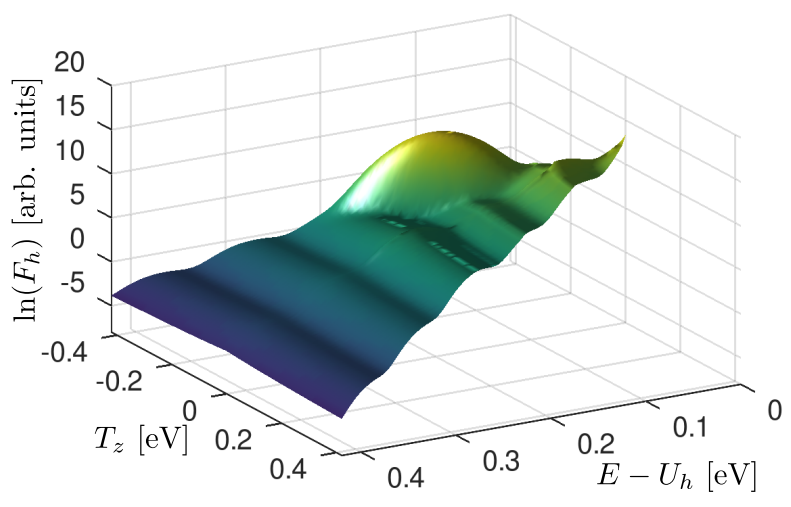

(c) Hole distribution at $U_{c}=0$

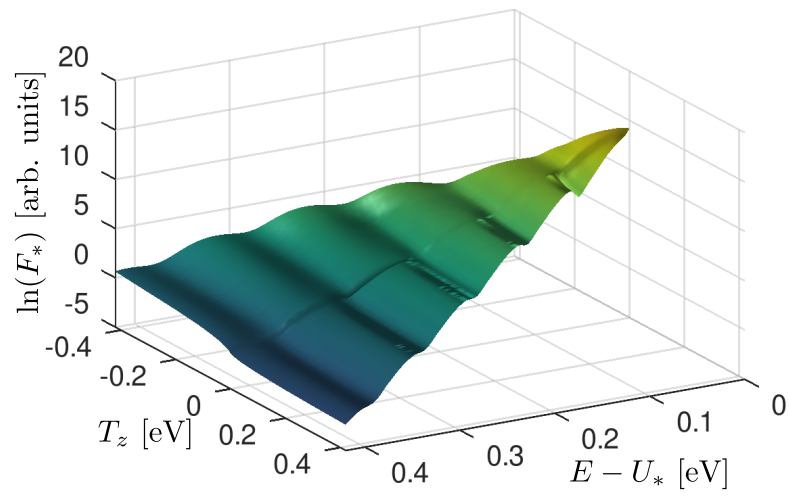

(b) Electron distribution at $U_{c}=21.4 \mathrm{meV}$

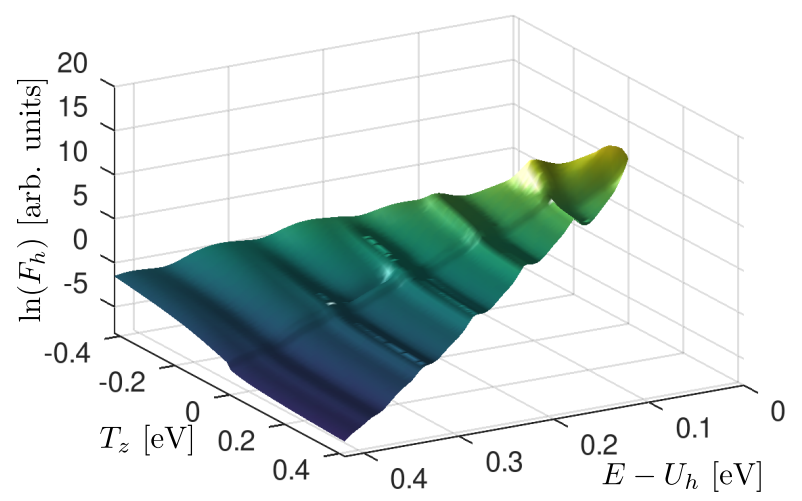

(d) Hole distribution at $U_{c}=21.4 \mathrm{meV}$

FIG. 3. Distribution functions for the injected electrons (upper panels) and holes (lower panels) for parameter set (a) at $U_{c}=0$, that is, at the interface and at $U_{c}=21.4 \mathrm{meV}$. Instead of $E$ and $T$ the variables $E-U_{s}$ and $T_{z}=E-U_{s}-T$ are used, where we attached to the latter a sign to denote the distributions of left- $\left(T_{z}<0\right)$ and right-moving $\left(T_{z}>0\right)$ particles. The injection peaks at $E-U_{*}=I_{*}^{\text {in }}=0.2 \mathrm{eV}$ and at $E-U_{h}=I_{h}^{\text {in }}=0.15 \mathrm{eV}$ are clearly visible in the data for $U_{c}=0$. Away from the interface, the peak gradually vanishes. Replicas due to phonon emission and absorption can be also seen, as well as the drop at $T_{z}=0$, signaling right-moving states to be less populated than left-moving ones. Colors are used only for visibility reasons and the triangular shape is due to energy conservation.

on the plasma side. It indicates that electrons and holes do not recombine spatially concurrently in our model.

The merging of the double layer with the bulk regions on either side of the interface and the working of the recombination process are shown in Fig. 6, where we plot, for both sides of the interface, as a function of $U_{c}$ the profiles of the carrier densities and fluxes. Recall, due to the coordinate transformation, the effectively infinite halfspaces in the spatial coordinate $z$ are mapped onto finite intervals on the $U_{c}$ axis. The embedding can be clearly seen in the upper panel. On the solid side, only for $U_{1} / 2<U_{c}<0$ is the electron density $n_{*}$ (blue) larger than the hole density $n_{h}$ (red), while for $U_{c}<U_{1} / 2$ the ordering is reversed. Taking the acceptor density $n_{A}$ into account, which balances the hole density due to doping but is not included in the plot to make the scales comparable, the quasi-neutral p-doped region emerges for $U_{c}$ approaching $U_{1}$. On the plasma side, on the other hand, ions (blue) dominate electrons (red) for
$0<U_{c}<U_{p}$, while for $U_{c} \approx U_{p}$ a quasi-neutral region appears merging, for $U_{c}$ approaching $U_{w}$, the negative sheath in front of the plasma source installed at $U_{c}=U_{w}$ by the Schwager-Birdsall construction.

The workings of the recombination process can be inferred from the flux profiles plotted in the lower panel of Fig. 6. Electron and ion fluxes are equal on the plasma side and continuously merge at $U_{c}=0$ with the electron and hole fluxes. From the flux continuity $j_{e}(0)=$ $j_{i}(0)=j_{*}(0)=j_{h}(0)$, the parameter $\alpha=n_{i}^{\mathrm{LM}} / n_{e}^{\mathrm{LM}}$, introduced in appendix $\mathrm{B}$ and characterizing the strength of the plasma source can be obtained. For the data shown in Fig. [6 we find $\alpha=11$. Due to electron-hole recombination inside the solid the fluxes decay. The hole flux decays faster than the electron flux, indicating that holes are destroyed closer to the interface than electrons. That the recombination of holes and electrons are spatially separated we have already noticed in the density profiles of Fig. 4. It can be explained by looking at the trap occu- 


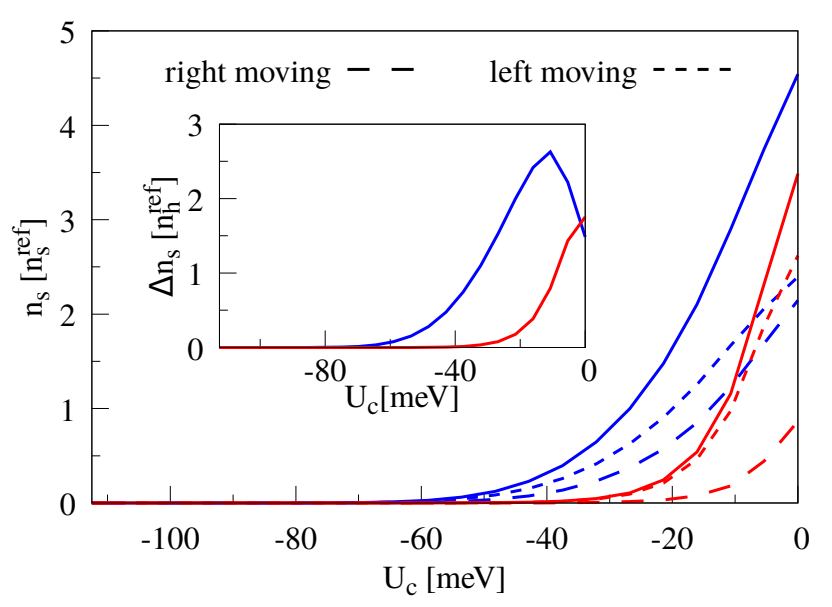

FIG. 4. Total (solid lines) and directional (long and short dashed lines) densities of the injected carriers for the parameter set (a). For plotting purposes we introduced reference densities $n_{*}^{r e f}=6 \cdot 10^{12} \mathrm{~cm}^{3}$ and $n_{h}^{r e f}=10^{12} \mathrm{~cm}^{3}$. The positive difference of left and right moving densities $\Delta n_{s}$, shown in the inset, is on the same order of magnitude for electrons and holes indicating that for both polarities surplus carriers move more likely to the left than to the right.

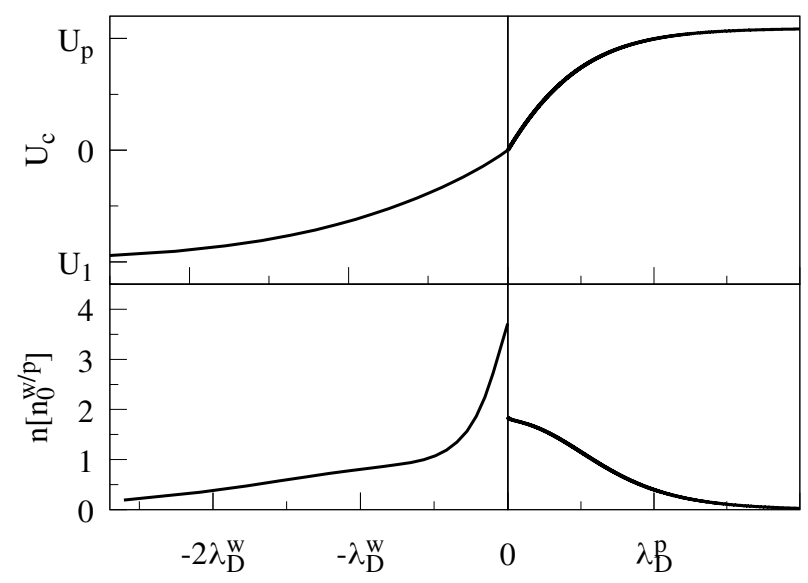

FIG. 5. Potential energy (upper panel) and net charge density (lower panel) for parameter set (a) as a function of $z$ in units of the Debye lengths, $\lambda_{D}^{w}=1.821 \mu \mathrm{m}$ and $\lambda_{D}^{p}=9.107 \mu \mathrm{m}$. The kink in $U_{c}$ at $z=0$ signals the matching condition (2). To fit the density profiles into a single plot, we scaled them on the solid side by $n_{0}^{w}=10^{13} \mathrm{~cm}^{-3}$ and on the plasma side by $n_{0}^{p}=-10^{12} \mathrm{~cm}^{-3}$.

pancy shown in the inset of Fig. 6. Holes have to recombine with an electron from the trap, that is, they require an occupied trap site, while electrons need empty trap sites. From the inset we see traps highly occupied close to the interface. Thus, in our model, holes preferentially recombine there, while electrons, requiring empty traps, have to move further into the solid, where the probability of finding them is higher.
In reality, the two fluxes should decay equally fast. That in our model this is not the case is due to the approximation we used to determine the trap occupancy. Instead of the full electron and hole distribution functions, we employed in A14 only the half-Maxwellians arising from the doping background. By neglecting the contributions of the injected carriers, which are small but nevertheless present, the trap occupancy is not determined selfconsistently. In the present formulation of our model, it can thus not react to the injected carriers. Inserting the full distributions, however, would have led to nonlinear collision integrals, artificially dominating the kinetics due to the high trap densities $N_{t}$ we have to use to ensure complete recombination in the part of the simulation domain which is numerically resolved. Since the kinetic scenario we wanted to develop-destruction of plasma flux impinging on a dielectric by electron-hole recombination inside it-is not affected by the inconsistency, we did not include this additional complexity into the model.

\section{CONCLUSION}

We have presented a selfconsistent kinetic model for the electric double layer at a dielectric plasma-solid interface that embraces plasma generation on one and plasma loss on the other side of the interface. Conduction band electrons and valence band holes are injected into the solid with unit probability for each impinging electron and ion. From the solid side, charge carriers cannot cross the interface which is thus modelled as a perfect absorber. Inside the solid electrons and holes scatter on optical phonons, leading to energy and momentum relaxation, before they recombine nonradiatively via traps in the energy gap of the dielectric. The microscopic picture encoded in our model is thus the one of a plasma source whose fluxes are equalized and balanced by the recombination of electron and hole fluxes in the space charge region of the solid.

Computational constraints in the numerical solution of the Boltzmann equation on the solid side forced us to treat charge injection by phenomenological source functions. The basic kinetics-injection of surplus charge carriers into the solid, followed by relaxation and recombination establishing a quasi-stationary double layer-is however still present in the simplified model. Based on an iterative scheme, geared towards solving Boltzmann equations with distribution functions specified at the endpoints of the integration domain, we presented the numerical solution of the kinetic equations, focusing in particular on the handling of singular points. A similar strategy could be applied on the plasma side in case it is made collisional.

Although quantitatively we cannot yet make hard predictions, because of the limitations of the phenomenological source functions, the perfect absorber assumption, and the idealistic treatment of the electronic struc- 


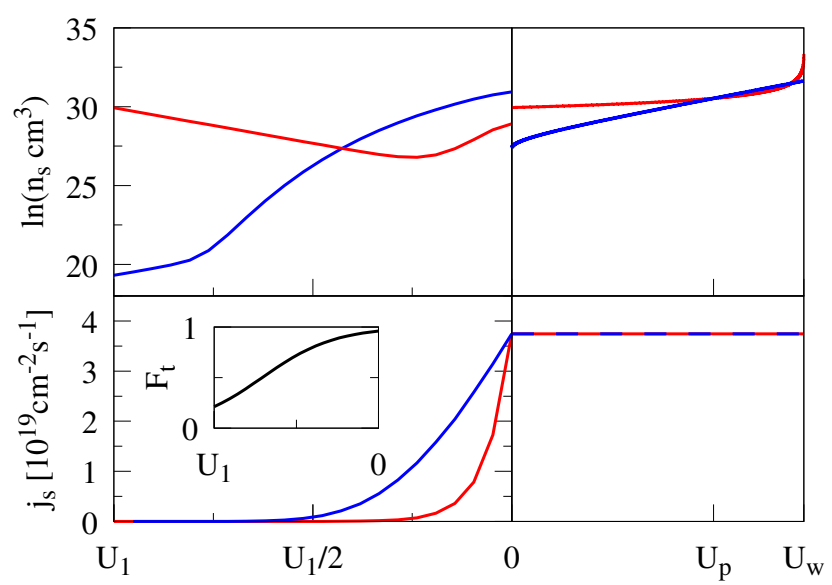

FIG. 6. Mobile charge carriers (upper panel) and the fluxes (lower panel) as a function of $U_{c}$ for parameter set (a). Data for electrons (holes, ions) are plotted in blue (red). Inside the solid, electrons prevail for $U_{1} / 2<U_{c}<0$, while for $U_{c}<$ $U_{1} / 2$ holes dominate, indicating the p-doped bulk. The quasineutral bulk plasma emerges on the other side of the interface for $U_{c} \approx U_{p}$. Also seen is the negative sheath in front of the plasma source at $U_{c}=U_{w}$. The electron and ion fluxes of the plasma merge at $U_{c}=0$ with the electron and hole fluxes of the solid, which then decay nonconcurrently due to the variation of the occupancy of the traps shown in the inset, placing empty (occupied) traps, required for electron (hole) recombination, further away from (closer to) the interface.

ture of the interface, which neglects, for instance, adsorbate layers likely to be present in a plasma environment, the numerical results show the feasibility of the scheme. From the distribution functions of the holes and the electrons we calculated the solid-bound density and potential profiles merging the plasma sheath from the solid side. Combined with approaches describing the merging of the sheath with the bulk plasma in more detail than we have done, taking, for instance, ion-neutral collisions into account, a complete picture of the double layer can thus be developed. The results demonstrate moreover that the charge kinetics inside the solid and the plasma can be treated on an equal footing, opening thus the door for a kinetic analysis of miniaturized semiconductor-based plasma devices combining gaseous and solid-state electronics.

\section{ACKNOWLEDGMENTS}

Support by Deutsche Froschungsgemeinschaft through project BR-1994/3-1 is greatly acknowledged.

\section{Appendix A: Collision integrals}

For the numerics it is convenient to split the collision integrals into an in- and an out-scattering part,

$$
I_{\text {coll }}^{\gtrless}=\Phi_{s}^{\gtrless}\left(U_{c}, E, T\right)-\gamma_{s}^{\gtrless}\left(U_{c}, E, T\right) F_{s}^{\gtrless}\left(U_{c}, E, T\right),
$$

defining implicitly the functions $\Phi_{s}^{\gtrless}$ and $\gamma_{s}^{\gtrless}$ entering the Boltzmann equation (6). Starting with the standard forms of the collision integrals it is straightforward to work out expressions for $\Phi_{s}^{\gtrless}$ and $\gamma_{s}^{\gtrless}$. Below we give them for the scattering processes included in this work: electron $(s=*)$ and hole $(s=h)$ scattering by polar optical phonons [33] and nonradiative electron-hole recombination via traps in the energy gap of the dielectric according to the Shockley-Read-Hall mechanism [34 36].

In the dilute limit, applicable to the situation we study, the collision integral for scattering by optical phonons becomes [33]

$$
\begin{aligned}
I_{s}^{\text {scat }}(z, \mathbf{k})=\int \frac{\mathrm{d}^{3} k^{\prime}}{(2 \pi)^{3}}\left[W\left(\mathbf{k}^{\prime}, \mathbf{k}\right) F_{s}\left(z, \mathbf{k}^{\prime}\right)\right. \\
\\
\left.\quad-W\left(\mathbf{k}, \mathbf{k}^{\prime}\right) F_{s}(z, \mathbf{k})\right],
\end{aligned}
$$

where the rate for scattering from $\mathbf{k}$ to $\mathbf{k}^{\prime}$ is given by

$$
\begin{aligned}
W\left(\mathbf{k}, \mathbf{k}^{\prime}\right)= & V \frac{2 \pi}{\hbar}\left|M\left(\left|\mathbf{k}-\mathbf{k}^{\prime}\right|\right)\right|^{2} \\
& \times\left[\left(1+n_{b}\right) \delta\left(E_{\mathbf{k}}-E_{\mathbf{k}^{\prime}}-\hbar \omega_{0}\right)\right. \\
& \left.\quad+n_{b} \delta\left(E_{\mathbf{k}}-E_{\mathbf{k}^{\prime}}+\hbar \omega_{0}\right)\right]
\end{aligned}
$$

with

$$
|M(q)|^{2}=\frac{\hbar \omega_{0}}{V} \frac{8 \pi q^{2} \tilde{\alpha} \hbar c}{\left(q^{2}+q_{s}^{2}\right)^{2}}\left(\frac{\varepsilon_{0}}{\varepsilon_{\infty}}-\frac{\varepsilon_{0}}{\varepsilon}\right)
$$

the square of the matrix element for electron (hole)phonon coupling. The standard notation is used throughout in the formulae, $E_{\mathbf{k}}$ is the kinetic energy of the electron (hole), $\hbar \omega_{0}$ is the energy of the (dispersionless, optical $)$ phonon, and $n_{b}=1 /\left(\exp \left(\hbar \omega_{0} / k_{B} T_{*}\right)-1\right)$ is the occupation number of the phonon. In the expression for the matrix element, $c$ is the vacuum speed of light, $\tilde{\alpha}$ is the fine structure constant, $q_{s}$ is a screening momentum, and $\varepsilon$ and $\varepsilon_{\infty}$ are the dielectric constants at low and high frequencies, respectively. In the atomic units used in the main text,

$$
\begin{aligned}
W\left(\mathbf{k}, \mathbf{k}^{\prime}\right)= & 16 W_{0} \frac{q^{2}}{\left(q^{2}+q_{s}^{2}\right)^{2}} \\
& \times\left[\left(1+n_{b}\right) \delta\left(E_{\mathbf{k}}-E_{\mathbf{k}^{\prime}}-\hbar \omega_{0}\right)\right. \\
& \left.\quad+n_{b} \delta\left(E_{\mathbf{k}}-E_{\mathbf{k}^{\prime}}+\hbar \omega_{0}\right)\right]
\end{aligned}
$$

with $W_{0}=4 \hbar \omega_{0}\left(1 / \varepsilon_{\infty}-1 / \varepsilon\right)$. Since the carrier concentrations are rather low, we neglect in the following the 
screening wave number $q_{s}$. Parts of the calculations can then be performed analytically.

The functions $\gamma_{s}^{\gtrless}\left(U_{c}, E, T\right)$ and $\Phi_{s}^{\gtrless}\left(U_{c}, E, T\right)$ appearing in the Boltzmann equation (6) are the integrals of either $W\left(\mathbf{k}, \mathbf{k}^{\prime}\right)$ or $W\left(\mathbf{k}^{\prime}, \mathbf{k}\right) F_{s}\left(z, \mathbf{k}^{\prime}\right)$ over $\mathbf{k}^{\prime}$. In the limit $q_{s}=0$, we find, after rewriting the momenta $\mathbf{k}$ and $\mathbf{k}^{\prime}$ in the coordinates $U_{c}, E, T, E^{\prime}$, and $T^{\prime}$, and distinguishing distributions for left- and right-moving particles,

$$
\gamma_{s}^{\gtrless}\left(U_{c}, E, T\right)=\frac{4 W_{0}}{v_{s}\left(U_{c}, E, 0\right)}\left[n_{b} \operatorname{arsinh}\left(\sqrt{\frac{E-U_{s}}{\hbar \omega_{0}}}\right)+\left(n_{b}+1\right) \operatorname{arsinh}\left(\sqrt{\frac{E-U_{s}}{\hbar \omega_{0}}-1}\right)\right],
$$

showing that this function is the same for both directions of motion, and

$$
\begin{aligned}
& \Phi_{s}^{\gtrless}\left(U_{c}, E, T\right)=W_{0} \times \\
& \sum_{ \pm}\left(n_{b}+\frac{1}{2} \pm \frac{1}{2}\right) \int_{0}^{E-U_{s} \pm \hbar \omega_{0}} \frac{\mathrm{d} T^{\prime}}{v_{s}\left(U_{s}, E \pm \hbar \omega_{0}, T^{\prime}\right)}\left[\frac{F_{s}^{\gtrless}\left(U_{c}, E \pm \hbar \omega_{0}, T^{\prime}\right)}{d_{-}\left(U_{c}, E, T, T^{\prime}, \pm \hbar \omega_{0}\right)}+\frac{F_{s}^{\lessgtr}\left(U_{c}, E \pm \hbar \omega_{0}, T^{\prime}\right)}{d_{+}\left(U_{c}, E, T, T^{\prime}, \pm \hbar \omega_{0}\right)}\right]
\end{aligned}
$$

with

$$
d_{ \pm}\left(U_{c}, E, T, T^{\prime}, \hbar \omega_{0}\right)=\left[\left(T+T^{\prime}+\left(\sqrt{E-U_{s}-T^{\prime}+\hbar \omega_{0}} \pm \sqrt{E-U_{s}-T}\right)^{2}\right)^{2}-4 T T^{\prime}\right]^{1 / 2}
$$

The second term of $\gamma_{s}^{\gtrless}$ and the in-scattering-byabsorption term in $\Phi_{s}^{\gtrless}$, that is, the term with the minus sign, only occurs for $E-U_{s}>\hbar \omega_{0}$. Note, the upper labels $\gtrless$ and $\lessgtr$ of the distributions on the right hand side of (A7) are independent of the \pm sign. They correspond to the $\gtrless$ of $\Phi_{s}^{\gtrless}$ on the left hand side of the equation.

We now turn to the kinetic formulation [34] of the Shockley-Read-Hall electron-hole recombination 35, 36. The collision integral coupling the trap occupancy $F_{t}$ with the electron distribution function $F_{*}^{\gtrless}$ reads

$$
I_{*}^{\mathrm{tr} \gtrless}=\left(1-F_{*}^{\gtrless}\right) \Gamma_{G}^{*} N_{t} F_{t},-F_{*}^{\gtrless} \Gamma_{R}^{*} N_{t}\left(1-F_{t}\right)
$$

while the one coupling $F_{t}$ it to the hole distribution function $F_{h}^{\gtrless}$ is

$$
I_{h}^{\mathrm{tr} \gtrless}=\left(1-F_{h}^{\gtrless}\right) \Gamma_{G}^{h} N_{t}\left(1-F_{t}\right)-F_{h}^{\gtrless} \Gamma_{R}^{h} N_{t} F_{t} .
$$

Therein $N_{t}$ is the trap density,

$$
\Gamma_{R}^{s}=\sigma_{s} v_{s}^{\text {tot }}
$$

is the recombination rate for species $s$, and

$$
\begin{aligned}
\Gamma_{G}^{*} & =\sigma_{*} v_{*}^{\text {tot }} \exp \left(\left(E_{t}-\left(E-U_{s}+E_{g}\right)\right) / k_{B} T_{*}\right), \\
\Gamma_{G}^{h} & =\sigma_{h} v_{h}^{\text {tot }} \exp \left(\left(-\left(E-U_{s}\right)-E_{t}\right) / k_{B} T_{h}\right)
\end{aligned}
$$

are the corresponding generation rates, where $\sigma_{s}$ is the capture cross section, $v_{s}^{\text {tot }}$ is the total velocity (not to be confused with $v_{s}\left(E, U_{c}, T\right)$ which is the velocity in $z$-direction, that is, $\left.v_{s}^{\text {tot }}=v_{s}\left(E, U_{c}, 0\right)\right)$, and $E_{t}$ is the energy level of the traps.
At quasi-stationarity, the trap occupancy is given by the detailed balance condition. Integrating A9 and (A10) over $E$ and $T$ and equating the results, yields

$$
\begin{aligned}
F_{t}\left(U_{c}\right)= & \left(m_{*} \int \frac{\mathrm{d} E \mathrm{~d} T}{v_{*}} \Gamma_{R}^{*} F_{*}^{>+<}\right. \\
& \left.+m_{h} \int \frac{\mathrm{d} E \mathrm{~d} T}{v_{h}} \Gamma_{G}^{h}\left(2-F_{h}^{>+<}\right)\right) \\
\times & \left(m_{*} \int \frac{\mathrm{d} E \mathrm{~d} T}{v_{*}}\left(\Gamma_{R}^{*} F_{*}^{>+<}+\Gamma_{G}^{*}\left(2-F_{*}^{>+<}\right)\right)\right. \\
+ & \left.m_{h} \int \frac{\mathrm{d} E \mathrm{~d} T}{v_{h}}\left(\Gamma_{R}^{h} F_{h}^{>+<}+\Gamma_{G}^{h}\left(2-F_{h}^{>+<}\right)\right)\right)^{-1}
\end{aligned}
$$

with $F_{s}^{>+<}$denoting $F_{s}^{>}\left(E, U_{c}, T\right)+F_{s}^{<}\left(E, U_{c}, T\right)$. In the detailed balance condition (A14), we account only for the charge carriers due to the doping, described by half-Maxwellian distribution functions. The surplus electrons and holes coming from the plasma affect the balance only weakly because of their low density. In leading approximation, they can thus be neglected.

Splitting (A9) and A10 into out- and in-scattering contributions and distinguishing distributions for leftand right-moving electrons and holes yields

$$
\begin{aligned}
\gamma_{*}^{\operatorname{tr}} & =\Gamma_{G}^{*} N_{t} F_{t}+\Gamma_{R}^{*} N_{t}\left(1-F_{t}\right), \\
\gamma_{h}^{\operatorname{tr}} & =\Gamma_{G}^{h} N_{t}\left(1-F_{t}\right)+\Gamma_{R}^{h} N_{t} F_{t}, \\
\Phi_{*}^{\operatorname{tr}} & =\Gamma_{G}^{*} N_{t} F_{t}, \\
\Phi_{h}^{\operatorname{tr}} & =\Gamma_{G}^{h} N_{t}\left(1-F_{t}\right),
\end{aligned}
$$

which after eliminating $F_{t}$ by using (A14) gives the form of the functions used in (6). 


\section{Appendix B: Plasma sheath}

To make the present work self-contained, we summarize in this appendix the formulae for the collisionless sheath forming on the plasma side of the interface. The merging of the plasma sheath-in the absence of collisions-with the bulk plasma is established by a construction due to Schwager and Birdsall [2]. It mimics the quasi-neutral, field-free bulk plasma by an inflection point at $U_{c}=U_{p}$ arising between the sheath at the interface at $U_{c}=0$ and the sheath in front of a plasma source imagined to sit at $U_{c}=U_{w}$.

The plasma source at $U_{c}=U_{w}$ ejects electrons and ions with the half-Maxwellian distributions (10). With the boundary condition $n_{s}\left(U_{w}\right)=n_{s}^{\mathrm{LM}}$ a trajectory analysis of the collisionless Boltzmann equations on the plasma side 21] leads to the density profiles

$$
\frac{n_{i}\left(U_{c}\right)}{n_{i}^{\mathrm{LM}}}=f(a)-\sqrt{\frac{a}{\pi}},
$$

where $a=\frac{U_{w}-U_{c}}{k_{B} T_{i}}$, and

$$
\frac{n_{e}\left(U_{c}\right)}{n_{e}^{\mathrm{LM}}}=e^{-\left(a^{\prime}+b\right)}\left[e^{b}-f(b)+\sqrt{\frac{b}{\pi}}\right]
$$

with $a^{\prime}=\frac{U_{w}-U_{c}}{k_{B} T_{e}}$ and $b=\frac{U_{c}}{k_{B} T_{e}}$. The function

$$
f(x)=\frac{1}{2} e^{x} \operatorname{erf}_{c}(\sqrt{x})+\sqrt{x / \pi}
$$

is connected to the complementary error function $\operatorname{erf}_{c}(x)=1-\operatorname{erf}(x)$. For the coordinate transformation (11) we need the integrals over the profiles given by

$$
\int_{U_{c}}^{U_{w}} \mathrm{~d} U \frac{n_{i}(U)}{n_{i}^{\mathrm{LM}} k_{B} T_{i}}=f(a)-\frac{1}{2}
$$

and

$$
\begin{aligned}
\int_{U_{c}}^{U_{w}} \mathrm{~d} U \frac{n_{e}(U)}{n_{e}^{\mathrm{LM}} k_{B} T_{e}}=1 & -e^{-a^{\prime}} \\
& +e^{a^{\prime}+b}[f(b)-f(a+b)] .
\end{aligned}
$$

To determine the inflection point at $U_{c}=U_{p}$, the conditions $\mathcal{E}\left(U_{p}\right)=0$ and $n\left(U_{p}\right)=0$ have to be worked out. Introducing $x_{y}=U_{x} / k_{B} T_{y}$ with $x \in\{p, w\}$ and $y \in\{i, e\}$ the condition of the vanishing electric field yields

$$
\frac{n_{i}^{\mathrm{LM}}}{n_{e}^{\mathrm{LM}}}=\frac{k_{B} T_{e}}{k_{B} T_{i}} \frac{1-e^{p_{e}-w_{e}}+e^{-w_{e}}\left[f\left(p_{e}\right)-f\left(w_{e}\right)\right]}{f\left(w_{i}-p_{i}\right)-\frac{1}{2}},
$$

whereas the vanishing of the net charge density becomes

$$
\begin{aligned}
\frac{n_{i}^{\mathrm{LM}}}{n_{e}^{\mathrm{LM}}}=\exp \left(p_{e}-w_{e}+p_{i}\right. & \left.-w_{e}\right) \\
& \times \frac{1+\Phi\left(\sqrt{p_{e}}\right)}{1-\Phi\left(\sqrt{w_{i}-p_{i}}\right)} .
\end{aligned}
$$

Augmenting Eqs. (B6) and (B7) with the flux balance (20), using

$$
j_{e}\left(U_{c}\right)=-n_{e}^{\mathrm{LM}} \sqrt{\frac{k_{B} T_{e}}{\pi}} e^{-w_{e}}
$$

and

$$
j_{i}\left(U_{c}\right)=-n_{i}^{\mathrm{LM}} \sqrt{\frac{k_{B} T_{i}}{m_{i} \pi}}
$$

to be obtained upon inserting (B1) and (B2) into (17), leads finally to three equations for the four unknowns $U_{w}, U_{p}, n_{\rho}^{\mathrm{LM}}$, and $n_{i}^{\mathrm{LM}}$. In the model of Schwager and Birdsall [2], which does not include the solid, only three of the parameters can thus be fixed. Considering the ratio $\alpha=n_{i}^{\mathrm{LM}} / n_{e}^{\mathrm{LM}}$ as the strength of the plasma source, $U_{w}, U_{p}$ and $\alpha$ are usually the parameters calculated. In our model, extending into the solid, the matching of the electric field (2) at $U_{c}=0$, that is, the charge neutrality of the double layer yields however an additional equation. At the end, we can thus determine all four parameters.

\section{Appendix C: Integration routines}

In this appendix we describe the integration routines used in the numerical treatment of Eqs. (25) and (26), focusing on the discretization and the handling of singular points.

The three-dimensional integration domain, spanned by the variables $U_{c}, E$, and $T$, is shown in Fig. 7 For all three the discretization step $\Delta$ is used, to be taken as a fraction of $\hbar \omega_{0}$. The potential energy $U_{c}$ ranges from $U_{1}$ to 0 , the total energy $E$ is at least $U_{s}$ and in principal unbound, and the lateral kinetic energy $T$ takes values from 0 to $E-U_{s}$. To keep the integration domain also in the variable $E$ finite, we use an energy cutoff $E_{\mathbf{k}}^{\text {cutoff }}=$ $0.4 \mathrm{eV}$ for the total kinetic energy $T+T_{z}$ of the charge carriers measured from the bottom of the bands.

By discretizing the integrals singular points have to be carefully treated. The square-root singularity due to the vanishing of $v_{s}\left(U_{c}, E, T\right)$ at $T=E-U_{s}$ can be removed by substitution. The $U_{c}$-integrals in Eqs. (25) and (26) are then solved in one step, without further interpolation points, by linearization. Some integrals are however still singular because $\xi_{s}$ is an exponential function of a possibly diverging integral. They have to be done by hand. Two types of integrals have to be distinguished: Integrals where $\mathcal{E}(U) \neq 0$ and integrals where $\mathcal{E}(U)=0$ which occur however only at the end point $U=U_{1}$.

First, we consider the case $\mathcal{E}(U) \neq 0$. The integrand in $\xi_{s}$ as well as the product of the functions in front of $\xi_{s}$, except of $v_{s}$, which has been removed by the substitution

$$
Z=\sqrt{U_{0} \pm U}
$$

where the $+\operatorname{sign}$ and $U_{0}=E-T+\chi$ is used for electrons, and the $-\operatorname{sign}$ and $U_{0}=E-T-E_{g}-\chi$ is used for holes, 


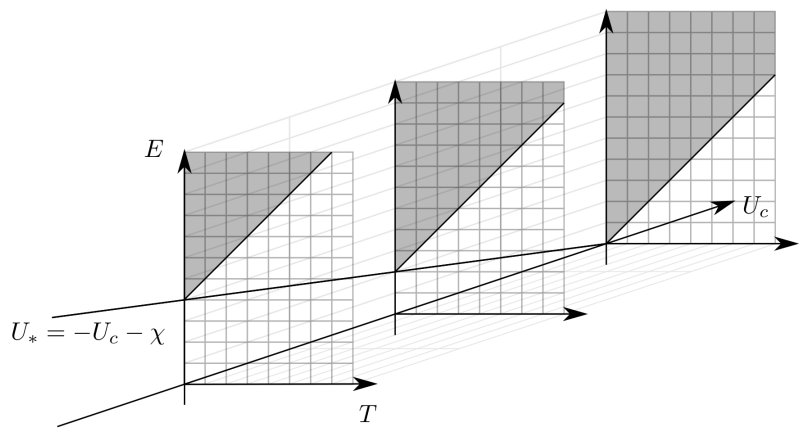

FIG. 7. Schematic illustration of the three-dimensional integration domain for conduction band electrons spanned by the variables $U_{c}, E$, and $T$. The domain is cut by the surface $v_{*}\left(U_{c}, E, T\right)=0$ leading to turning points at $T=E-U_{*}\left(U_{c}\right)$ separating the region where electrons are allowed to move shown in dark $\left[T<E-U_{*}\left(U_{c}\right)\right]$ from the forbidden region $\left[T>E-U_{*}\left(U_{c}\right)\right]$. For the valence band holes the domain is divided by the function $T=E-U_{h}\left(U_{c}\right)$ giving rise to a different shape. The elementary discretization step, to be the same in all three dimensions, $\Delta=\hbar \omega_{0} / n$ with $\hbar \omega_{0}$ the phonon energy. The energy cutoff $E_{\mathbf{k}}^{\text {cutoff }}=N \hbar \omega_{0}$ is used for the total kinetic energy $T+T_{z}$ of the carriers measured from the bottom of the bands, limiting thereby the variable $E$. For the results presented in this work we typically used $n=8$, $N=14$ yielding around $100^{3}$ discretization points.

can be linearized. Then, integrals of the form

$$
\int_{Z_{0}}^{Z_{1}} \mathrm{~d} Z\left(f_{0}+Z f_{1}\right) \exp \left(-\left(g_{0}+Z g_{1}\right)\left(Z_{1}-Z\right)\right)
$$

appear for Eq. (25), while integrals of the type

$$
\int_{Z_{0}}^{Z_{1}} \mathrm{~d} Z\left(f_{0}+Z f_{1}\right) \exp \left(-\left(g_{0}+Z g_{1}\right)\left(Z-Z_{0}\right)\right)
$$

are found for Eq. (26). The abbreviations, subsuming numerical coefficients arising from the linearization, should be clear from the context. For instance, $f_{0}=$ $\Phi_{s}^{\gtrless}\left(U_{c}\right) /\left(\mathcal{E}\left(U_{c}\right) v_{s}\left(U_{c}\right)\right)$. Using the identities

$$
\int \mathrm{d} x \exp \left(a x+b x^{2}\right)=\frac{1}{2} \sqrt{\frac{\pi}{b}} \exp \left(-\frac{a^{2}}{4 b}\right) \operatorname{erfi}\left(\frac{a+b x}{2 \sqrt{b}}\right)
$$

and

$$
\begin{array}{r}
\int \mathrm{d} x x \exp \left(a x+b x^{2}\right)=\frac{1}{2 b} \exp \left(a x+b x^{2}\right) \\
-\frac{1}{4} \frac{a}{b} \sqrt{\frac{\pi}{b}} \exp \left(-\frac{a^{2}}{4 b}\right) \operatorname{erfi}\left(\frac{a+b x}{2 \sqrt{b}}\right),
\end{array}
$$

the integrals can be related to the imaginary error function erfi $(x)$ to be calculated as follows: For positive $b$ we use the Dawson function $F(x)=\sqrt{\pi} \exp \left(-x^{2}\right) \operatorname{erfi}(x) / 2$ while for negative $b$ we employ the identity erfi(ix) $=$ $\operatorname{ierf}(x)$. The error function erf $(x)$ in turn is evaluated by routines of standard libraries. For large arguments, where the routines have problems, we expand erf $(x)$ together with the factor $\exp \left(-a^{2} / 4 b\right)$ into a power series.

To deal with the integrals where $\mathcal{E}=0$, we assumed and verified a posteriori, that $\mathcal{E}$ starts linearly with $U_{c}$. The divergence of $1 / \mathcal{E}$ in the integral of the exponent of $\xi_{s}$ is then cancelled by the same divergence in the $U_{c^{-}}$ integrals of (25) and (26). A linear approximation for $\mathcal{E}\left(U_{c} \gtrsim U_{1}\right)$ together with (C1) implies $\mathcal{E}(Z) \sim Z^{2}-Z_{0}^{2}$. Linearizing the remaining parts of the integrands relates the integrals to the incomplete beta function,

$$
B_{x}(\alpha+1,1-\beta)=\int_{0}^{x} \mathrm{~d} y \frac{y^{\alpha}}{(1-y)^{\beta}},
$$

or-in case $Z_{0}=0$ (that is, for $T=E-U_{s}$ )-to the incomplete gamma function

$$
\Gamma(a, x)=\int_{0}^{1 / x} \mathrm{~d} y y^{-a-1} \exp \left(-\frac{1}{y}\right) .
$$

The parameters $a, \alpha, \beta$ and $Z_{0}$, again numerical coefficients arising from the linearization, can be straightforwardly albeit tediously determined. Depending on the arguments, the beta function is evaluated either in terms of a continued fraction representation or in terms of the hypergeometric function, using

$$
B_{x}(a, b)=\frac{x^{a}}{a}{ }_{2} F_{1}(a, 1-b, a+1, x) .
$$

Likewise, the incomplete gamma function is obtained from a continued fraction expansion in cases where the evaluation with routines from standard libraries fails.

After the integrals have been evaluated in the form just described we have an algebraic set of equations which can be iterated in the three-dimensional domain shown in Fig. 7 The particular shape of the domain depends on the species through the function $U_{s}$. We found convergence to be reached faster if the iteration process does not destroy detailed balance in the phonon collision integrals. We thus put-for phonon collisions only-in (25) and (26) the term $-\gamma_{s}^{\gtrless} F_{s}^{\gtrless}$ into the function $\Phi_{s}^{\gtrless}$. 
[1] I. Langmuir and H. Mott-Smith, Gen. Electr. Rev. 27, 449 (1924).

[2] L. A. Schwager and C. K. Birdsall, Phys. Fluids B 2, 1057 (1990).

[3] K.-U. Riemann, J. Phys. D: Appl. Phys. 24, 493 (1991).

[4] R. N. Franklin, J. Phys. D: Appl. Phys. 36, R309 (2003).

[5] R. P. Brinkmann, J. Phys. D: Appl. Phys. 42, 194009 (2009).

[6] S. Robertson, Plasma Phys. Control. Fusion 55, 093001 (2013).

[7] P. N. Hu and S. Ziering, Phys. Fluids 9, 2168 (1966).

[8] F. Taccogna, S. Longo, and M. Capitelli, Phys. Plasma 11, 1220 (2004).

[9] D. Sydorenko, I. D. Kaganovich, Y. Raitses, and A. Smolyakov, Phys. Rev. Lett. 103, 145004 (2009).

[10] J. P. Sheehan, N. Hershkowitz, I. D. Kaganovich, H. Wang, Y. Raitses, E. V. Barnat, B. R. Weatherford, and D. Sydorenko, Phys. Rev. Lett. 111, 075002 (2013).

[11] S. Langendorf and M. Walker, Phys. Plasma 22, 033515 (2015).

[12] M. D. Campanell and M. V. Umansky, Phys. Rev. Lett. 116, 085003 (2016).

[13] R. N. Franklin, Plasma phenomena in gas discharges (Clarendon Press, Oxford, 1976).

[14] A. Marcak, C. Corbella, T. de los Arcos, and A. von Keudell, Rev. Sci. Instrum. 86, 106102 (2015).

[15] V. I. Demidov, S. F. Adams, I. D. Kaganovich, M. E. Koepke, and I. P. Kurlyandskaya, Phys. Plasma 22, 104501 (2015).

[16] M. Daksha, B. Berger, E. Schuengel, I. Korolov, A. Derzsi, M. Koepke, Z. Donkó, and J. Schulze, J. Phys. D: Appl. Phys. 49, 234001 (2016).

[17] F. X. Bronold and H. Fehske, Phys. Rev. Lett. 115, 225001 (2015).

[18] M. Pamperin, F. X. Bronold, and H. Fehske, Plasma Sources Sci. Technol. 27, 084003 (2018).

[19] M. Daksha, A. Derzsi, Z. Mujahid, D. Schulenberg, B. Berger, Z. Donkó, and J. Schulze, Plasma Sources Sci. Technol. 28, 034002 (2019).

[20] R. L. Heinisch, F. X. Bronold, and H. Fehske, Phys. Rev. B 85, 075323 (2012).

[21] F. X. Bronold and H. Fehske, J. Phys. D: Appl. Phys. 50, 294003 (2017).

[22] M. Bonitz, A. Filinov, J.-W. Abraham, K. Balzer,
H. Kählert, E. Pehlke, F. X. Bronold, M. Pamperin, M. Becker, D. Loffhagen, and H. Fehske, Front. Chem. Sci. Eng. 13, 201 (2019).

[23] S. Arumugam, M. Perumal, K. P. Anjana, S. V. M. Satyanarayna, and S. K. Sinha, Phys. Plasma 27, 023512 (2020).

[24] N. P. Ostrom and J. G. Eden, Appl. Phys. Lett. 87, 141101 (2005).

[25] R. Dussart, L. J. Overzet, P. Lefaucheux, T. Dufour, M. Kulsreshath, M. A. Mandra, T. Tillocher, O. Aubry, S. Dozias, P. Ranson, J. B. Lee, and M. Goeckner, Eur. Phys. J. D 60, 601 (2010).

[26] P. A. Tchertchian, C. J. Wagner, T. J. Houlahan Jr., B. Li, D. J. Sievers, and J. G. Eden, Contr. Plasma Phys. 51, 889 (2011).

[27] M. K. Kulsreshath, L. Schwaederle, L. J. Overzet, P. Lefaucheux, J. Ladroue, T. Tillocher, O. Aubry, M. Woytasik, G. Schelcher, and R. Dussart, J. Phys. D: Appl. Phys. 45, 285202 (2012).

[28] M. Tabib-Azar and P. Pai, Micromachines 8, 117 (2017).

[29] J. G. Eden, S.-J. Park, J. H. Cho, M. H. Kim, T. J. Houlahan, B. Li, E. S. Kim, T. L. Kim, S. K. Lee, K. S. Kim, J. K. Yoon, S. H. Sung, P. Sun, C. M. Herring, and C. J. Wagner, IEEE Trans. Plasma Sci. 41, 661 (2013).

[30] T. E. Sheridan and J. Goree, Phys. Fluids B 3, 2796 (1991).

[31] K.-U. Riemann, J. Phys. D: Appl. Phys. 36, 2811 (2003).

[32] Z. Sternovsky, Plasma Sources Sci. Technol. 14, 32 (2005).

[33] B. K. Ridley, Quantum processes in semiconductors (Clarendon Press, Oxford, 1999).

[34] K. Rupp, C. Jungemann, S.-M. Hong, M. Bina, T. Grasser, and A. Jüngel, J. Comput. Electron. 15, 939 (2016).

[35] R. N. Hall, Phys. Rev. 87, 228 (1951).

[36] W. Shockley and W. T. Read, Phys. Rev. 87, 835 (1952).

[37] A. A. Grinberg and S. Luryi, Solid-St. Electron. 35, 1299 (1992).

[38] M. M. Dignam and A. A. Grinberg, Phys. Rev. B 50, 4345 (1994).

[39] A. R. St. Denis and D. L. Pulfrey, J. Appl. Phys. 84, 4959 (1998).

[40] K. Konistis and Q. Hu, J. Appl. Phys. 91, 5400 (2002). 